\title{
Synthesis and characterization of some new Schiff bases of 2-oxonaphtho[2,1-b][1,4]oxazine
}

\author{
Pankaj Chovatiya, Charmy Mehta, Hardik Senjani, Anamik Shah, \\ Hitendra S. Joshi* \\ Department of Chemistry, Saurashtra University, Rajkot - 360 005, Gujarat, India \\ *E-mail address: drhsjoshi49@gmail.com
}

\begin{abstract}
The literature review reveal that $[1,4]$-oxazine derivatives represent one of the most active classes of compounds possessing wide spectrum of biodynamic activities and use as potent therapeutic agents. In the present work, a series of Schiff base of 2-(2,3-dihydro-2-oxonaphtho[2,1b][1,4]oxazin-1-yl)acetohydrazide, $\mathbf{5 a - 5 j}$ has been synthesized using 1-aminonaphthalen-2-ol. Various aromatic aldehyde were react with carbohydrazide $\mathbf{4}$ in the presence of acid to produce the 2oxonaphtho[2,1-b][1,4]oxazin Schiff base derivatives with good yields. All synthesized compounds were characterized by IR, NMR and Mass spectrometry analysis.
\end{abstract}

Keywords: 2-Oxonaphtho[2,1-b][1,4]oxazine; Schiff-base

\section{INTRODUCTION}

The [1,4]-oxazine scaffold is a structural subunit of many naturally occurring and synthetic bioactive compounds. Derivatives of $[1,4]$-oxazine moiety have found wide biological activities such as antiulcer [1], antihypertensive [2], antifungal [3], anticancer [4] and anti-thromobotic [5]. A new, selective and atom-economical synthetic methodologies for the synthesis of functionalized 2-oxonaphtho[2,1-b][1,4]oxazine starting from simple building blocks through an ordered sequence of steps is of particular interest also in view of the wide range of biological activities shown by many derivatives of these classes of heterocycles [6]. Herein, we studied the synthesis Schiff base [7-14] of 2-(2,3-dihydro-2oxonaphtho[2,1-b][1,4] oxazin-1-yl)acetohydrazide $\mathbf{4}$ series begins with commercially available 1-aminonaphthalen-2-ol.

Target compounds 5a-5j were synthesized according to Scheme 1. 1H-naphtho[2,1b][1,4] oxazin-2-one 2 was prepared from 1-aminonaphthalen-2-ol hydrochloride as starting material, which reacted with 2-chloroacetyl chloride [15]. Compound 4 was synthesized by 1H-naphtho[2,1-b][1,4]oxazin-2-one 2 and ethylbromoacetate followed by reaction with hydrazine hydrate. Compounds $\mathbf{5} \mathbf{a}-\mathbf{5 j}$ were obtained through the condensation reaction of compound 4 with different substituted aromatic aldehyde. The further work including development of new compound series and biological activity is underway in our laboratory. 


\section{EXPERIMENTAL}

All chemicals and solvents were purchased from Spectrochem Pvt Ltd., Mumbai of AR grade and were used without further purification. Melting points were taken in open capillary method and are uncorrected. IR spectra were recorded on FTIR-8400 spectrophotometer (Shimadzu, Kyoto, Japan), using DRS probe KBr pallet. ${ }^{1} \mathrm{H}-\mathrm{NMR}$ spectra of the synthesized compounds were recorded on a Bruker-Avance-II (400 MHz) DMSO- $d_{6}$ solvent.

Chemical shifts are expressed in $\delta$ ppm downfield from TMS as an internal standard. Mass spectra were determined using direct inlet probe on a GCMS-QP 2010 mass spectrometer (Shimadzu, Kyoto, Japan). Physical constants of the synthesized compounds $\mathbf{5 a - 5 j}$ are shown in Table 1.

\section{1. Synthesis of 2-chloro-N-(2-hydroxynaphthalen-1-yl)-acetamide (1)}

To the suspended solution of 1-aminonaphthalen-2-ol hydrochloride $(6 \mathrm{mmol})$ in THF $(10 \mathrm{ml})$; triethylamine $(2 \mathrm{ml})$ and chloroacetyl chloride $(6 \mathrm{mmol})$ was added under cooling condition and allowed to stir overnight at room temperature. The reaction mixture was poured onto ice-cold water and extracted with ethyl acetate. The obtained organic layer was washed with water and saturated brine, and dried over anhydrous sodium sulfate. The solvent was evaporated; titled compound was obtained as a dark brown solid. Yield: $86 \%$

\section{2. Synthesis of $1 H-n a p h t h o[2,1-b][1,4]$ oxazin-2-one (2)}

In the next step, compound 1 (5 mmol) was dissolved in $10 \mathrm{ml} \mathrm{DMF}$, to this potassium carbonate $(0.8 \mathrm{~g})$ and sodium iodide (catalytic amount) were added at room temperature. The reaction mixture was refluxed for $4 \mathrm{~h}$ at $80{ }^{\circ} \mathrm{C}$ and was poured onto crushed ice, and extracted with ethyl acetate.

The obtained organic layer was washed successively with $1 \mathrm{~N}$ hydrochloric acid, water and saturated brine, and dried over anhydrous sodium sulfate. The solvent was evaporated; title compound was obtained as a dark brown solid. Yield: $78 \%$

\section{3. Synthesis of ethyl 2-(2,3-dihydro-2-oxonaphtho[2,1-b][1,4]oxazin-1-y1)acetate (3)}

To a solution of compound 2 ( $6 \mathrm{mmol})$ in $10 \mathrm{ml}$ DMF, potassium carbonate $(0.9 \mathrm{~g})$ was added at room temperature. This was followed by the addition of Ethylbromoacetate (6 mmol) at $0-5{ }^{\circ} \mathrm{C}$. The reaction mixture was refluxed until completion of reaction. The reaction mixture cooled to room temperature and poured onto ice-cold water followed by extraction with ethyl acetate. The combined organic layer was evaporated to get compound $\mathbf{3}$ as brown liquid, which was directly taken for hydrolysis. Crude yield $64 \%$

\section{4. Synthesis of 2-(2,3-dihydro-2-oxonaphtho[2,1-b][1,4]oxazin-1-yl)acetohydrazide (4)}

To a solution of compound 3 ( $4 \mathrm{mmol})$ in ethanol $(5 \mathrm{ml})$, hydrazine hydrate was added dropwise at $0-5{ }^{\circ} \mathrm{C}$. Reaction mass was stirred at room temperature (about $4 \mathrm{~h}$ ). On cooling precipitates were filtered, dried and purified by crystallization. Yield: $68 \%$

\section{5. General procedure for the synthesis of Schiff base of 2-(2,3-dihydro-2-oxonaphtho [2,1-b][1,4]oxazin-1-yl)acetohydrazides (5a-5j)}

A mixture of 4 ( $1 \mathrm{mmol})$ and substituted benzaldehyde $(1 \mathrm{mmol})$ in presence of catalytic amount of acetic acid was refluxed with stirring until the reaction got complete (reaction 
progress was monitored by TLC). The mixture was then poured on crushed ice. The solid product was separated by filtration, dried and purified by crystallization. Yield: $61 \%$ to $87 \%$

\section{REACTION SCHEME}<smiles>Nc1c(O)ccc2ccccc12</smiles><smiles>O=C(CCl)Nc1c(O)ccc2ccccc12</smiles>

1

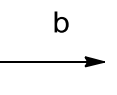<smiles>O=C1COc2ccc3ccccc3c2N1</smiles>

2 C<smiles>[R]c1ccc(/C=N/NC(=O)CN2C(=O)COc3ccc4ccccc4c32)cc1</smiles>

5a-j<smiles>NNC(=O)CN1C(=O)COc2ccc3ccccc3c21</smiles><smiles>CCOC(=O)CN1C(=O)COc2ccc3ccccc3c21</smiles>

3

Scheme 1. (a) triethylamine \& chloroacetyl chloride; (b) DMF, $\mathrm{K}_{2} \mathrm{CO}_{3}$, \& NaI; (c) DMF, ethylbromo acetate; (d) $\mathrm{MeOH}, \mathrm{NH}_{2} \mathrm{NH}_{2} \cdot \mathrm{H}_{2} \mathrm{O}$; (e) methanol, Substituted Aldehyde, acetic acid

Table 1. Physical constant of 2-(2,3-dihydro-2-oxonaphtho[2,1-b][1,4]oxazin-1-yl) acetohydrazide derivatives $(5 \mathrm{a}-5 \mathrm{j})$.

\begin{tabular}{|c|c|c|c|c|c|c|}
\hline No & Comp. & $\mathbf{R}$ & $\begin{array}{c}\text { Molecular } \\
\text { Formula }\end{array}$ & $\begin{array}{c}\text { Molecular } \\
\text { Weight }\end{array}$ & $\begin{array}{c}\text { Yield } \\
\text { (\%) }\end{array}$ & $\begin{array}{c}\text { M.P. } \\
\left({ }^{\circ} \mathbf{C}\right)\end{array}$ \\
\hline 1 & $5 \mathrm{a}$ & $2-\mathrm{OCH}{ }_{3}$ & $\mathrm{C}_{22} \mathrm{H}_{19} \mathrm{~N}_{3} \mathrm{O}_{4}$ & 389 & 65 & $188-190$ \\
\hline 2 & $5 \mathrm{~b}$ & $4-\mathrm{F}$ & $\mathrm{C}_{21} \mathrm{H}_{16} \mathrm{FN}_{3} \mathrm{O}_{3}$ & 377 & 73 & $141-143$ \\
\hline 3 & $5 \mathrm{c}$ & $3-\mathrm{Br}$ & $\mathrm{C}_{21} \mathrm{H}_{16} \mathrm{BrN}_{3} \mathrm{O}_{3}$ & 438 & 64 & $211-214$ \\
\hline 4 & $5 \mathrm{~d}$ & $4-\mathrm{Cl}$ & $\mathrm{C}_{21} \mathrm{H}_{16} \mathrm{ClN}_{3} \mathrm{O}_{3}$ & 393 & 68 & $202-204$ \\
\hline 5 & $5 \mathrm{e}$ & $3,4-\mathrm{di}-\mathrm{OCH} \mathrm{CH}_{3}$ & $\mathrm{C}_{23} \mathrm{H}_{21} \mathrm{~N}_{3} \mathrm{O}_{5}$ & 419 & 82 & $252-253$ \\
\hline 6 & $5 \mathrm{f}$ & $4-\mathrm{OCH}$ & $\mathrm{C}_{22} \mathrm{H}_{19} \mathrm{~N}_{3} \mathrm{O}_{4}$ & 389 & 73 & $247-249$ \\
\hline 7 & $5 \mathrm{~g}$ & $4-\mathrm{Me}$ & $\mathrm{C}_{22} \mathrm{H}_{19} \mathrm{~N}_{3} \mathrm{O}_{3}$ & 373 & 80 & $197-198$ \\
\hline 8 & $5 \mathrm{~h}$ & $2-\mathrm{Cl}$ & $\mathrm{C}_{21} \mathrm{H}_{16} \mathrm{ClN}_{3} \mathrm{O}_{3}$ & 393 & 74 & $221-224$ \\
\hline 9 & $5 \mathrm{i}$ & $4-\mathrm{OH}$ & $\mathrm{C}_{21} \mathrm{H}_{17} \mathrm{~N}_{3} \mathrm{O}_{4}$ & 375 & 61 & $217-218$ \\
\hline 10 & $5 \mathrm{j}$ & $4-\mathrm{Br}$ & $\mathrm{C}_{21} \mathrm{H}_{16} \mathrm{BrN}_{3} \mathrm{O}_{3}$ & 438 & 87 & $245-246$ \\
\hline
\end{tabular}




\section{SPECTRAL DATA}

4. 1. (E)-N'-(2-methoxybenzylidene)-2-(2-oxo-2,3-dihydro-1H-naphtho[2,1b][1,4]oxazin1-yl)acetohydrazide Compound 5a

$\mathrm{Mp} 188-190{ }^{\circ} \mathrm{C}$; IR $(\mathrm{KBr}) v\left(\mathrm{~cm}^{-1}\right): 3073,3031,2957,2847,1625,1462,1442,1325,1258$, $1140,1065,1018,825,748,701,685 \mathrm{~cm}^{-1} ;{ }^{1} \mathrm{H}$ NMR (DMSO- $\left.d_{6}, 400 \mathrm{MHz}\right): \delta(\mathrm{ppm}) 8.36(\mathrm{~s}$, $1 \mathrm{H}), 8.20-8.11(\mathrm{~m}, 1 \mathrm{H}), 8.02(\mathrm{~s}, 1 \mathrm{H}), 7.95(\mathrm{dd}, 1 \mathrm{H}), 7.75(\mathrm{dd}, 1 \mathrm{H}), 7.54-7.39(\mathrm{~m}, 4 \mathrm{H})$, $7.36-7.23(\mathrm{~m}, 2 \mathrm{H}), 7.14(\mathrm{dd}, 1 \mathrm{H}), 4.98(\mathrm{~s}, 2 \mathrm{H}), 4.78(\mathrm{~s}, 2 \mathrm{H}), 4.44(\mathrm{~s}, 3 \mathrm{H}) ; \mathrm{M}^{+}(\mathrm{m} / \mathrm{z})=389$; Elemental Analysis for $\mathrm{C}_{22} \mathrm{H}_{19} \mathrm{~N}_{3} \mathrm{O}_{4}$ : Calculated C (67.86 \%); H $(4.92 \%)$; N (10.79\%); Found: C (67.25\%); H (4.78 \%); N (10.02\%).

\section{2. (E)-N'-(4-fluorobenzylidene)-2-(2-oxo-2,3-dihydro-1H-naphtho[2,1-b][1,4]oxazin-1- yl)acetohydrazide Compound $5 b$}

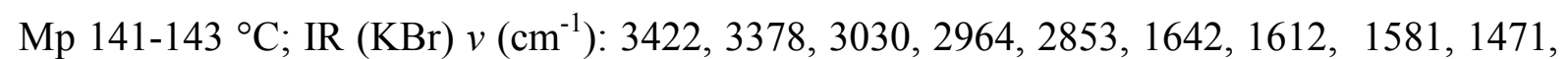
$1368,1247,1156,1057,1014,819,744,710,678 \mathrm{~cm}^{-1} ;{ }^{1} \mathrm{H}$ NMR (DMSO- $\left.d_{6}, 400 \mathrm{MHz}\right): \delta$ (ppm) $8.13(\mathrm{~s}, 1 \mathrm{H}), 8.03(\mathrm{~s}, 1 \mathrm{H}), 8.00-7.90(\mathrm{~m}, 2 \mathrm{H}), 7.84-7.71(\mathrm{~m}, 3 \mathrm{H}), 7.50-7.39$ (m, $2 \mathrm{H}), 7.36-7.23(\mathrm{~m}, 3 \mathrm{H}), 4.81(\mathrm{~s}, 2 \mathrm{H}), 4.49(\mathrm{~s}, 2 \mathrm{H}) ; \mathrm{M}^{+}(\mathrm{m} / \mathrm{z})=377$; Elemental Analysis for $\mathrm{C}_{21} \mathrm{H}_{16} \mathrm{FN}_{3} \mathrm{O}_{3}$ : Calculated C (66.84\%); H (4.27\%); N (11.14\%); Found: $\mathrm{C}(66.39 \%) ; \mathrm{H}$ (4.12\%); N (10.89\%)

\section{3. (E)-N'-(3-bromobenzylidene)-2-(2-oxo-2,3-dihydro-1H-naphtho[2,1-b][1,4]oxazin- 1-yl)acetohydrazide Compound $5 \mathrm{c}$}

$\operatorname{Mp~211-214~}{ }^{\circ} \mathrm{C}$; IR (KBr) $v\left(\mathrm{~cm}^{-1}\right): 3063,2962,2854,1603,1545,1542,1452,1325,1260$, $1146,1060,1023,812,754,662,518 \mathrm{~cm}^{-1}$; ${ }^{1} \mathrm{H}$ NMR (DMSO-d, $\left.400 \mathrm{MHz}\right): \delta(\mathrm{ppm}) 8.45(\mathrm{~s}$, $1 \mathrm{H}), 8.13-8.03(\mathrm{~m}, 1 \mathrm{H}), 8.03(\mathrm{~s}, 1 \mathrm{H}), 7.99-7.90(\mathrm{~m}, 2 \mathrm{H}), 7.75(\mathrm{dd}, 1 \mathrm{H}), 7.70-7.55(\mathrm{~m}$, 2H), $7.50-7.38(\mathrm{~m}, 3 \mathrm{H}), 7.33(\mathrm{~d}, 1 \mathrm{H}), 4.81(\mathrm{~s}, 2 \mathrm{H}), 4.49(\mathrm{~s}, 2 \mathrm{H}) ; \mathrm{M}^{+}(\mathrm{m} / \mathrm{z})=438$; Elemental Analysis for $\mathrm{C}_{21} \mathrm{H}_{16} \mathrm{BrN}_{3} \mathrm{O}_{3}$ : Calculated $\mathrm{C}(57.55 \%) ; \mathrm{H}(3.68 \%)$; N (9.59\%); Found: C (57.22\%); H (3.48\%); N (9.39\%)

\section{4. (E)-N'-(4-chlorobenzylidene)-2-(2-oxo-2,3-dihydro-1H-naphtho[2,1-b][1,4]oxazin-1- yl)acetohydrazide Compound 5d}

Mp 202-204 ${ }^{\circ} \mathrm{C}$; IR (KBr) $v\left(\mathrm{~cm}^{-1}\right): 3080,2983,2867,1629,1572,1525,1462,1381,1245$, $1196,1046,1011,830,778,701,665,578 \mathrm{~cm}^{-1} ;{ }^{1} \mathrm{H}$ NMR (DMSO- $\left.d_{6}, 400 \mathrm{MHz}\right): \delta(\mathrm{ppm})$ $8.25(\mathrm{~s}, 1 \mathrm{H}), 8.13-8.04(\mathrm{~m}, 1 \mathrm{H}), 8.02(\mathrm{~s}, 1 \mathrm{H}), 8.00-7.90(\mathrm{~m}, 2 \mathrm{H}), 7.86-7.71(\mathrm{~m}, 3 \mathrm{H}), 7.51$ - $7.39(\mathrm{~m}, 4 \mathrm{H}), 7.33(\mathrm{~d}, 1 \mathrm{H}), 4.82(\mathrm{~s}, 2 \mathrm{H}), 4.49(\mathrm{~s}, 2 \mathrm{H}) ; \mathrm{M}^{+}(\mathrm{m} / \mathrm{z})=393$; Elemental Analysis for $\mathrm{C}_{21} \mathrm{H}_{16} \mathrm{ClN}_{3} \mathrm{O}_{3}$ : Calculated C (64.05\%); H (4.09\%); N (10.67\%); Found: C (63.86 \%); H $(4.05 \%) ; \mathrm{N}(10.58 \%)$

\section{CONCLUSION}

We have established facile and convenient method for the synthesis of Schiff base of 2-(2,3-dihydro-2-oxonaphtho[2,1-b][1,4]oxazin-1-yl)acetohydrazide under a conventional reagent. All synthesized compounds were obtained in good to moderate yield. All synthesized compounds were characterized by IR, NMR and Mass spectrometry analysis and are incorporate with the structure of compounds $\mathbf{5 a - 5} \mathbf{j}$. 


\section{ACKNOWLEDGMENT}

Authors are thankful to Department of Chemistry for providing laboratory facilities. Authors are also thankful to National Facility for Drug Discovery (NFDD) Saurashtra University, Rajkot for providing ${ }^{1} \mathrm{H}$ NMR spectral analysis of the compounds.

\section{References}

[1] Y. Katsura, S. Nishino, H. Takasugi, Chem. Pharm. Bull. 11 (1991) 2937.

[2] N. Kajino, Y. Shibouta, K. Nishikawa, K. Meguro, Chem. Pharm. Bull. 11 (1991) 2896.

[3] R. Fringuelli, D. Pietrella, F. Schiaffella, A. Guarraci, S. Perito, F.Bistoni, A.Vecchiarelli, Bioorg. Med. Chem. 10 (2002) 1681.

[4] M. G. Nair, O. C. Salter, R. L. Kisliuk, Y. Gaumont, J. Med. Chem. 26 (1983) 1164.

[5] B. O. Buckman, R. Mohan, S. Koovakkat, Bioorg. Med. Chem. Lett. 8 (1998) 2235.

[6] E. Blattes, B. Lockhart, P. Lestage, L. Schwendimann, P. Gressens, M. B. Fleury, M. Largeron, J. Med. Chem. 48 (2005) 1282.

[7] K. M. Thaker, V.V Kachhadia, H.S. Joshi, Indian Journal Of Chemistry Section B, 42(6) (2003) 1544.

[8] Nirav M. Shah, Hitendra S. Joshi, International Letters of Chemistry, Physics and Astronomy 6 (2014) 56-60.

[9] Piyush B. Vekariya, Jalpa R. Pandya, Vaishali Goswami, Hitendra S. Joshi, International Letters of Chemistry, Physics and Astronomy 7 (2014) 45-52.

[10] Prakash Mehta, Prakash Davadra, Nirav Shah, Hitendra Joshi, International Letters of Chemistry, Physics and Astronomy 10 (2014) 74-80.

[11] Prakash Mehta, Prakash Dawedra, Vaishali Goswami, Hitendra S. Joshi, International Letters of Chemistry, Physics and Astronomy 11(1) (2014) 1-8.

[12] S. M. S. Shariar, M. Jesmin, M. M. Ali, International Letters of Chemistry, Physics and Astronomy 7 (2014) 53-61.

[13] Mele Jesmin, M. Khairul Islam, Shaikh M. Mohsin Ali, International Letters of Chemistry, Physics and Astronomy 8 (2014) 64-72.

[14] Mallikarjun S. Yadawe, Shrishila N. Unki, Sangamesh A. Patil, International Letters of Chemistry, Physics and Astronomy 12 (2013) 94-104.

[15] Hirata Kazuyuki, et al. From PCT Int. Appl., 2007139002, 06 Dec 2007. 